\section{Submucous resection as an outpatient procedure}

Dear Sir,

We read with interest the paper by Buckley et al. entitled 'Submucous resection of the nasal septum as an outpatient procedure' (July 1991).

A comparison was made of submucous resection of the nasal septum (SMR) in two groups of patients. In one, the operation was performed under local anaesthesia as an outpatient procedure; in the other group, general anaesthesia was used, and the nose was packed for $24 \mathrm{~h}$ postoperatively. Although it was not stated, the assumption is that these patients remained in hospital for at least $24 \mathrm{~h}$ postoperatively.

We have performed a study of 53 consecutive SMR procedures under general anaesthesia. A standard Killian SMR technique was used, and the mucoperichondrial flaps were sutured together using a continuous $4 / 0$ Vicryl or catgut suture. Nasal packing was required in only four patients, the packs being removed after one hour. The mean time from the end of the operation to discharge from hospital was $146 \mathrm{mins}$ (range 80-220 minutes). Two patients were readmitted for treatment of reactionary haemorrhage. No patient developed a complication related to the general anaesthesia.

In our series, patients occupied a bed in the day surgery unit for either a morning or afternoon, thus permitting two patients to be treated per bed per day.

One of the advantages of general anaesthesia for SMR is that it facilitates the performance of other procedures at the same time, such as nasal polypectomy and submucous diathermy of inferior turbinates. Buckley et al. found that twice as many patients, 28 (56 per cent) underwent additional procedures in the general anaesthesia compared to the local anaesthesia group, 14 (28 per cent).

We agree that SMR can be performed under local anaesthesia, but feel that general anaesthesia is safe, quick, well tolerated, and permits discharge from hospital usually within two to three hours from surgery. It facilitates training of junior staff, and is particularly appropriate when other procedures need to be performed in addition to SMR.

Yours faithfully,

Theo Joseph, F.R.C.S.,

Senior Registrar in Otolaryngology,

Radcliffe Infirmary,

Oxford

Nicholas J. Marks, M.S., F.R.C.S.,

Consultant Otolaryngologist,

Royal Berkshire Hospital,

Reading.

Reply:

Dear Sir,

We read with interest the letter from Messrs Joseph and Marks.

Of course we are well aware that an SMR can be adequately performed under general anaesthetic and combined with other procedures as a day case. We have been operating in the fashion over the past two years with success. The point of our paper was to show that an SMR can also be performed adequately under local anaesthetic in an Outpatient Department. The relative costs of performing an SMR under different anaesthetic conditions was given in our paper.

We would like to stress that there are patients requiring an SMR that can be managed successfully and safely without having to resort to a general anaesthetic or the need of a day case theatre facility.

Yours faithfully,

A. F. Fitzgerald O'Connor F.R.C.S., Consultant ENT Surgeon,

St. Thomas' Hospital, London SE1 7EH. 